\title{
Estrategia de cualificación de los factores de investigación para la optimización de trabajos de investigación científica en las universidades privadas de Ica - 2014
}

\author{
Factors Qualification Strategy of Research for the Optimization in Scientific \\ Research at Privates Universities in Ica - 2014
}

\author{
Carlos Blanco Contreras ${ }^{1}$
}

http://dx.doi.org/10.21503/CienciayDesarrollo.2015.v18i1.04

\section{RESUMEN}

Para desarrollar un trabajo de investigación requiere sumar varios componentes como la política de estado en ciencia, tecnología e investigación, factores institucionales y el fenotipo de los docentes investigadores.

Las dos primeras variables han sido analizadas con mucha amplitud por expertos en estos temas mientras que la variable fenotipo (sexo, edad, formación, centro laboral) no han sido tomados en cuenta, es la razón del interés de este trabajo.

Para este propósito se ha utilizado un instrumento de medición de coeficiente intelectual utilizando el Test Progresiva de Raven, el que se ha aplicado a 210 docentes, y el análisis de los resultados indica que el 50\% de docentes que trabajaron en el periodo académico 2014 -I tienen un coeficiente intelectual superior al término medio, destacando el sexo femenino, los que ostentan grado académico de magíster y/o doctor, los de formación profesional en Ingeniería, Arquitectura y Salud, los que trabajan en la Facultad de Ingeniería-Salud, pero lo preocupante es que no hay trabajo de investigación visible.

Palabras clave: investigación del fenotipo del docente.

\section{ABSTRACT}

To develop a research project requires adding several components as state policy on science, technology and research, institutional factors and phenotype of educational researchers. The two first variables have been discussed widely by experts on these topics while the phenotype variable (sex, age, education, workplace) have not been taken into account, it is the reason for the interest in this work.

For this purpose, we used a measuring instrument of intelligence quotient using Raven's Progressive IQ; which has been applied to 210 teachers, and analysis of the results indicates that $50 \%$ of teachers who worked in the academic year 2014-I have an IQ higher than average, highlighting the female, those holding academic degree of master and/or doctor, the professional training Engineering, Architecture and Health, those working in the Faculty of Engineering - Health, but the worrying thing is that there isn't research work visible.

Key words: investigation of the phenotype of the teacher.

1 Doctor en Educación. Magíster en Docencia Universitaria y magíster en Sanidad Ambiental de la UV-UIMP, España. Ingeniero químico, especialista en monitoreo de calidad ambiental en la UNAM. Director de la E.A.P. de Ingeniería Ambiental, filial Ica. E-mail: proambiente2012@gmail.com 


\section{INTRODUCCIÓN}

La orientación adecuada de la investigación científica y tecnológica ha contribuido enormemente a posibilitar la elevación de la calidad de vida humana.

Este aspecto positivo de la ciencia es particularmente importante para un país como el nuestro, donde, en el presente, tenemos que importar desde el trigo, alimento básico del pueblo, hasta los ingredientes para elaborar detergentes. El Perú cuenta con suficientes recursos naturales y humanos como para iniciar un proceso de grandes transformaciones económicas y sociales que se traduzcan en mejores niveles de vida para todos sus habitantes.

En el 2010 se han censado 100 universidades, con un considerable crecimiento de las universidades privadas, éstas ascienden a un total de 65 , aumentando en 1,8 veces su número respecto a 1996.

Menos de la mitad de docentes han realizado investigaciones $(42,8 \%)$ en los dos últimos años. De estos docentes la mayor parte (el 61,7\%) ha realizado solo una investigación, el 26,0\% han realizado dos investigaciones y el $12,3 \%$ tres investigaciones. Los docentes de las universidades públicas desarrollan más investigaciones que sus pares de las universidades privadas $(62,5 \%$ y $31,5 \%$, respectivamente); la tendencia del número de investigaciones que realizan los docentes es similar en las universidades públicas como privadas.

De las diez áreas de conocimiento en los que desarrollan las investigaciones los docentes, las de mayor frecuencia son: Ingeniería y Tecnologías (21,2\%), Ciencias de la Salud y Psicología (16,0 $\%)$, Educación (15,4\%), Economía, empresariales y afines $(12,2 \%)$ y Ciencias Sociales y Humanidades $(12,0 \%)$.
Las universidades privadas de Ica (UPI) tienen una plana docente multidisciplinaria con formación y experiencia importante que bien puede contribuir con el desarrollo de trabajos de investigación en beneficio de la región y el Perú.

\section{HIPÓTESIS}

La estrategia de cualificación de los factores de investigación, como el fenotipo del docente, factores institucionales, y la política de Estado en Ciencia, Tecnología e Innovación (CTI), determinaría en la optimización de los trabajos de investigación en la universidades privadas de Ica.

\section{METODOLOGÍA DE LA INVESTIGACIÓN}

\section{Tipo y nivel de la investigación}

\section{a) Tipo de la investigación}

La investigación es de tipo teórica; se centra planteando una estrategia de cualificación de docentes considerando las características del docente investigador y las condiciones necesarias para hacer investigación.

\section{b) Nivel de la investigación}

El nivel de la investigación es multiparadigmática: descriptiva y explicativa.

\section{Método y diseño de la investigación}

\section{a) Método de investigación}

El instrumento que se utilizó es el test de inteligencia no verbal denominado el test progresivo de Raven.

Este test se aplicará a la muestra obtenida para este caso 210 docentes que trabajan en las universidades privadas. Para el análisis se tomará en cuenta el género, grado académico, formación, centro laboral y edad. 


\section{b) Diseño de la investigación}

La investigación corresponde al diseño no experimental, transversal.

La estrategia diseñada en el trabajo de investigación tiene una correlación entre sus variables pues a mayor uso de los factores indicados se logrará optimizar los trabajos de investigación.

\section{Población y muestra}

\section{a) Población}

Está constituida por los 465 docentes que trabajan en las 03 universidades privadas de Ica, 14 escuelas académicas profesionales para el periodo 2014 - I.

\section{b) Muestra}

Es una parte representativa de la población, que se ha determinado mediante la fórmula estadística

$$
n=\frac{Z^{2} \cdot P \cdot Q \cdot N}{e^{2} \cdot(N-1)+Z^{2} \cdot P \cdot Q}
$$

\section{Nomenclatura}

\begin{tabular}{lll}
\hline $\mathrm{N}$ & 465 & $\begin{array}{l}\text { Población de docentes } \\
\mathrm{p}\end{array}$ \\
$\mathrm{q}$ & 0.5 & $\begin{array}{l}\text { Proporción de población con } \\
\text { las características }\end{array}$ \\
$\mathrm{q}=1-\mathrm{p}$ & 0.5 & $\begin{array}{l}\text { Proporción de población sin } \\
\text { las características }\end{array}$ \\
$\mathrm{Z}^{2}$ & $(1.96)^{2}$ & Nivel de confianza \\
$\mathrm{E}=(5 \%)^{2}$ & $(0.05)^{2}$ & Error deseado por uno \\
\hline & $\mathrm{n}=210$ docentes
\end{tabular}

Técnicas e instrumentos de recolección de datos

\section{a) Técnicas}

Información indirecta. Recopilación de la información existente en fuentes bibliográfi- cas y estadísticas; en libros, revistas especializadas, artículos y publicaciones de expertos por Internet, trabajos de investigación anteriores, empleo del Internet y otros.

Información directa. Se obtendrá mediante la aplicación de encuestas en las muestras determinadas de las poblaciones consideradas, cuyas muestras serán obtenidas aleatoriamente en forma estratificada, de acuerdo a la proporción del número total de individuos por cada grupo poblacional citado.

\section{b) Instrumentos de recolección de datos}

\section{Ficha de investigación}

Se utilizaron las fichas de resumen, las fichas bibliográficas y las fichas textuales, para registrar la información que nos permitió elaborar el Marco Teórico y el Marco Conceptual del trabajo de investigación.

\section{Cuestionario}

Se aplica el Test Progresivo de Raven a los 210 docentes

\section{Fichas de observación}

Se elaborarán para conocer los aportes a la estrategia de cualificación de los directivos, expertos, docentes y estudiantes universitarios.

\section{c) Técnicas de procesamiento de los datos}

Para el procesamiento de datos, se realizarán las siguientes operaciones:

Clasificación. Para especificar criterios de agrupación de los datos obtenidos, determinándose las analogías correspondientes como: sexo, edad, formación, grado académico y facultad en la que enseña.

Codificación. Comprenderá la asignación de valores, a las preguntas y alternativas para conceder los puntajes respectivos 
Tabulación. Se refiere al conteo y sumatoria de la información obtenida

d) Técnicas de análisis e interpretación de la información

\section{Técnica de análisis}

Existen varios métodos para probar el coeficiente intelectual de las personas, pero esta vez se usa el Test Progresivo de Raven.

\section{Interpretación de la información}

Para la interpretación del Test Progresivo de Raven se seguirán estos pasos:

\section{- Corregir la prueba:}

Se trata de evaluar el acierto o el error en la solución propuesta por el docente en cada problema que plantea el test.

La corrección se realizará de dos maneras:

- Hojas de respuestas autocopiativas, utilizando un software, lo que facilita una co- rrección rápida y precisa sin necesidad de plantilla de corrección.

- Corrección mecanizada a través de lectora óptica, útil para evaluación de grandes grupos de sujetos.

\section{- Obtener el puntaje:}

Consiste en sumar el número de ítems acertados.

La puntuación directa máxima que se puede obtener es 60 puntos (escala general)

- Convertir el puntaje en percentil:

Calcular la puntuación del docente en relación con un grupo de 100 docentes con un rango de edad; consultando para ello una tabla.

\section{- Convertir el percentil en rango:}

Se trata de calificar con un índice ordinal la capacidad intelectual del docente.

Estos serían los 5 rangos principales:

\section{Tabla 1. Rango intelectual}

\begin{tabular}{|c|c|c|}
\hline PERCENTIL & $\begin{array}{c}\text { CAPACIDAD INTELECTUAL } \\
\text { IGUALADA O SOBREPASADA } \\
\text { EN EL GRUPO DE SU EDAD }\end{array}$ & RANGO INTELECTUAL \\
\hline $950+$ & $5 \%$ de los sujetos & I: Superioridad intelectual \\
\hline 90 & $10 \%$ de los sujetos & II: Superior al término medio \\
75 & $25 \%$ de los sujetos & \\
\hline 50 & $50 \%$ de los sujetos & III: Término medio \\
\hline 25 & $75 \%$ de los sujetos & IV: Inferior al término medio \\
10 & $90 \%$ de los sujetos & \\
\hline 5 & $95 \%$ de los sujetos & V: Deficiencia intelectual \\
\hline
\end{tabular}

Fuente: http://www.psicotecnicostest.com/testpruebaspsicometricas.asp 


\section{RESULTADOS}

La muestra consta de 210 docentes, de los cuales 73 corresponden al sexo femenino, que representa el $35 \%$, y 137 del sexo masculino, que representa el $65 \%$.

\section{Tabla 2. \% Docentes por sexo}

\begin{tabular}{rcc} 
SEXO & $\%$ & CANTIDAD \\
\hline Femenino & 35 & 73 \\
\hline Masculino & 65 & 137 \\
\hline
\end{tabular}

Fuente propia
También se distingue docentes desde 23 a 70 años de edad, siendo los de 41-50 el grupo de mayor cantidad, y el de 61-70 el menor.

\section{Tabla 3. Docentes por edad}

\begin{tabular}{|cc}
\hline EDAD & CANTIDAD \\
\hline $23-30$ & 23 \\
$31-40$ & 43 \\
$41-50$ & 78 \\
$51-60$ & 46 \\
$61-70$ & 20 \\
\hline
\end{tabular}

Fuente propia

\section{Coeficiente intelectual de todos los docentes en general}

La tabla 4 resume el número de docentes en los distintos rangos de coeficiente intelectual En la tabla 4 se presenta los valores de los percentiles y su respectivo diagnóstico de la capacidad

Tabla 4. Coeficiente intelectual

\begin{tabular}{ccccccccc} 
NORMA & $\mathbf{N}^{\circ}$ & $\mathbf{N}^{\circ}$ ORDEN & $\mathbf{K}_{\mathrm{j}}$ & INTERV & $\mathrm{f}$ & $\mathrm{P}_{\mathrm{i}}$ & RANGO & $\begin{array}{c}\text { COEFICIENTE } \\
\text { INTELECTUAL }\end{array}$ \\
P99 & 210 & 208 & 58 & $57-58$ & 12 & 99 & & \\
P95 & 210 & 200 & 57 & $56-57$ & 12 & 95 & $\mathrm{I}$ & $\mathrm{S}$ \\
P90 & 210 & 190 & 56 & $54-55$ & 31 & 90 & II+ & STM \\
P75 & 210 & 156 & 54 & $52-53$ & 51 & 75 & II & STM \\
P50 & 210 & 105 & 52 & $47-51$ & 54 & 50 & III+ & TM \\
P25 & 210 & 53 & 47 & $43-46$ & 30 & 25 & IV+ & ITM \\
P10 & 210 & 21 & 43 & $38-42$ & 10 & 10 & IV & ITM \\
P5 & 210 & 11 & 38 & $23-36$ & 10 & 5 & V & D \\
\hline
\end{tabular}

Fuente propia

intelectual (coeficiente intelectual) de los docentes del conjunto de los docentes que constituyen la muestra; de ella se observa que 24 docentes respondieron entre 56-58 preguntas correctas, de los 60 que corresponden al test de Raven, colocándose en un percentil 95 (P95), lo que quiere decir que hay un $5 \%$ de docentes con superioridad intelectual $(S)$ y estarían en el ran- go I. Así mismo 31 docentes respondieron 5455 preguntas correctas, colocándose en el P 90; quiere decir que hay un $10 \%$ de docentes con capacidad intelectual por encima de superior al término medio (STM) y estaría en el rango II+, después vemos que hay 51 docentes que respondieron 52-53 preguntas correctas, colocándose en el P75, quiere decir que hay $25 \%$ de docentes 
con capacidad intelectual (CI) superior al término medio (STM) y estaría en el rango II. Luego vemos en el cuadro 54 docentes que respondieron 47-51 preguntas correctas, colocándose en el P50, lo que quiere decir que hay $50 \%$ de docentes con capacidad intelectual (CI) igual a inferior del término medio (TM) y estaría en el rango III.

La figura 1 nos muestra la relación entre el número de docentes (f) que está representado con los números del eje horizontal con los percentiles $(\mathrm{Pi})$ que muestra en la parte superior de cada barra.

En cambio, la figura 2 muestra la relación entre las dos anteriores más el rango, que está

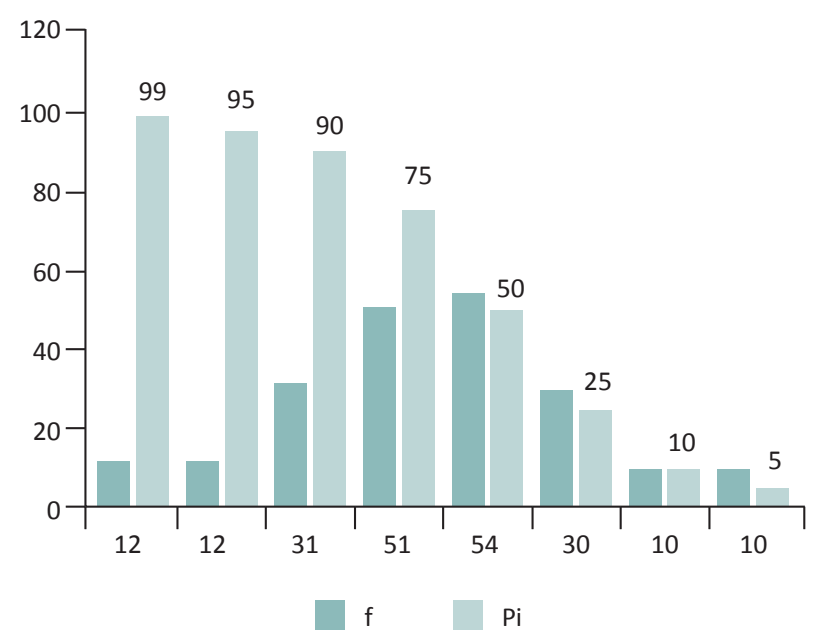

Figura 1. Relación de frecuencia (f) y percentil (Pi) docentes en general

Fuente propia

expresada con números romanos en el eje horizontal.

La figura 3 nos representa el \% de los docentes según su coeficiente intelectual (C.I), donde se destaca que hay un $50 \%$ de docentes que tienen un CI superior al termino medio (STM).

Para el análisis de este trabajo se agrupan en 04 grupos de coeficiente intelectual (C.I):

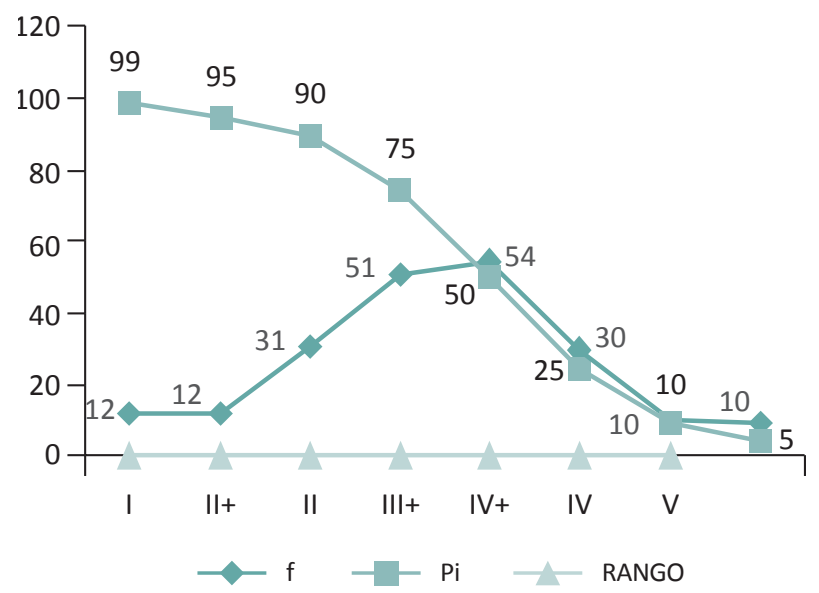

Figura 2. Relación f, Pi, $\mathrm{R}$ docentes en general. Fuente propia

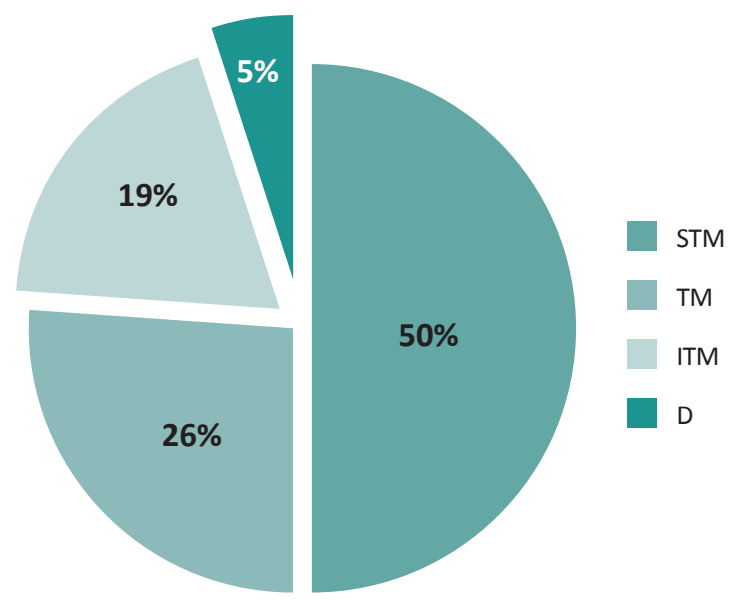

Figura 3. Porcentaje docentes en general según Fuente propia su C.I.

- STM = Superior al término medio que incluye al C.I. superior, que involucra los rangos II, II+, I.

- $\mathrm{TM}=$ Término medio, involucra III, III+.

- ITM = Inferior al término medio, involucra los rangos IV+, IV.

- $\mathrm{D}=$ Deficiente, involucra al rango $\mathrm{V}$.

I. A partir de aquí se muestran los resultados de los coeficientes intelectuales por cada fenotipo que se ha considerado para esta investigación. 
a) Comparación del coeficiente intelectual de los docentes por sexo

La figura 4 nos indica lo siguiente:

Tabla 5. Comparación del C.I. por sexo

\begin{tabular}{lrrrr} 
RAVEN & S. FEM. & $\%$ & S. MASC. & $\%$ \\
STM & 52 & 44 & 57 & 42 \\
TM & 19 & 26 & 42 & 31 \\
\hline ITM & 18 & 25 & 31 & 23 \\
D & 4 & 5 & 7 & 5 \\
\hline & 73 & 100 & 137 & 100 \\
\hline
\end{tabular}

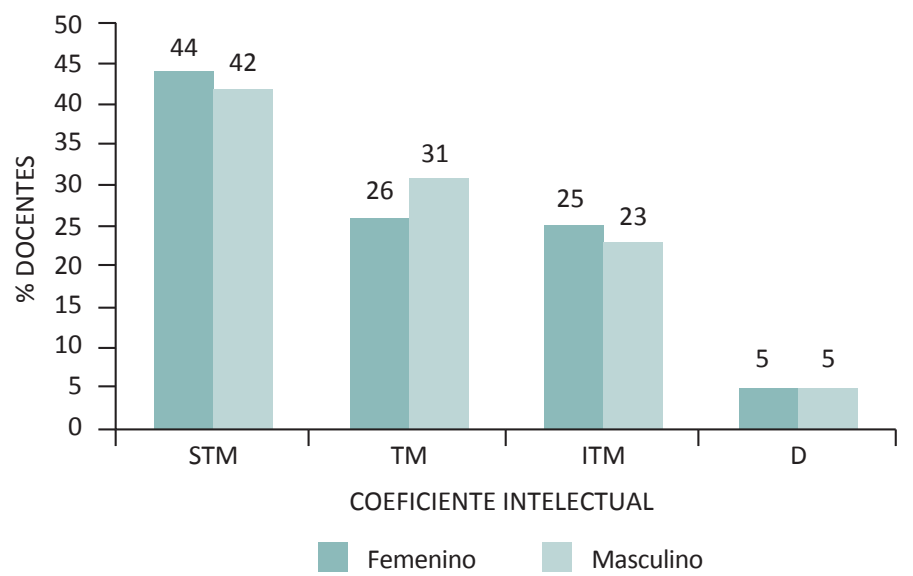

Figura 4.

- Las docentes del sexo femenino tienen mayor C.I. STM respecto al docente del sexo masculino en una proporción de $44 \%$ a $42 \%$ respectivamente.

- Los docentes del sexo masculino tienen mayor C.I. TM, respecto al docente del sexo femenino, en una proporción de $31 \%$ a $26 \%$ respectivamente.
- Las docentes del sexo femenino tienen mayor C.I. ITM, respecto al docente del sexo masculino, en una proporción de $25 \%$ a $23 \%$ respectivamente.

- Los docentes del sexo femenino como masculino tienen un porcentaje de $5 \%$ de docentes con C.I. D.

\section{b) Comparación del coeficiente intelectual de los docentes por su nivel académico}

Tabla 6. Comparación C.I. por su nivel académico

\begin{tabular}{lcrrr} 
RAVEN & $\begin{array}{c}\text { CON } \\
\text { GRADO }\end{array}$ & \multicolumn{1}{c}{$\%$} & $\begin{array}{c}\text { CON } \\
\text { TITULO }\end{array}$ & \multicolumn{1}{c}{$\%$} \\
STM & 36 & 46 & 50 & 45 \\
TM & 22 & 28 & 37 & 28 \\
\hline ITM & 16 & 21 & 26 & 20 \\
D & 4 & 5 & 9 & 7 \\
\hline & 78 & 100 & 132 & 100 \\
\hline
\end{tabular}

Fuente propia

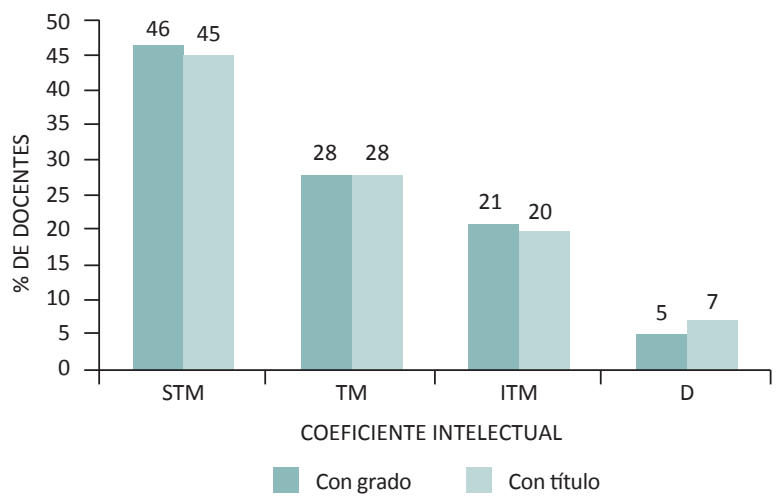

Figura 5. 
En la figura 5 se presentan los resultados obtenidos por los docentes en el test de Matrices Progresivas Raven escala general; aplicado a ambos sexos pero tomando en cuenta su nivel de formación (docentes titulados, docentes con grado de magíster y/o doctor)

- Los docentes con grado magíster y/o doctor tienen mayor C.I. STM, respecto al docente con solo título en una proporción de $46 \%$ a $45 \%$ respectivamente.
- Los docentes con solo título y grado de magíster y/o doctor tienen el mismo C.I. TM, de $28 \%$.

- Las docentes con grado de magister y/o doctor tienen un C.I. ITM mayor al docente con solo título, en una proporción de $21 \%$ a $20 \%$ respectivamente.

- Las docentes con solo título tienen un C.I. D mayor al docente con grado de magister y/o doctor, en una proporción de $5 \%$ a $7 \%$ respectivamente.

\section{c) Comparación del coeficiente intelectual de los docentes por facultad (entorno laboral)}

\section{Tabla 7. Comparación del C.I de docentes por facultades}

\begin{tabular}{lrrrrrr} 
RAVEN & F. ING Y ARQ & \multicolumn{1}{c}{$\%$} & F. C. DE LA SALUD & $\%$ & F. C. EMPR + DER & $\%$ \\
\hline STM & 42 & 46 & 36 & 45 & 17 & 44 \\
TM & 21 & 23 & 19 & 24 & 11 & 28 \\
\hline ITM & 23 & 25 & 21 & 26 & 9 & 23 \\
D & 5 & 5 & 4 & 5 & 2 & 5 \\
\hline & 91 & 100 & 80 & 100 & 39 & 100 \\
\hline
\end{tabular}

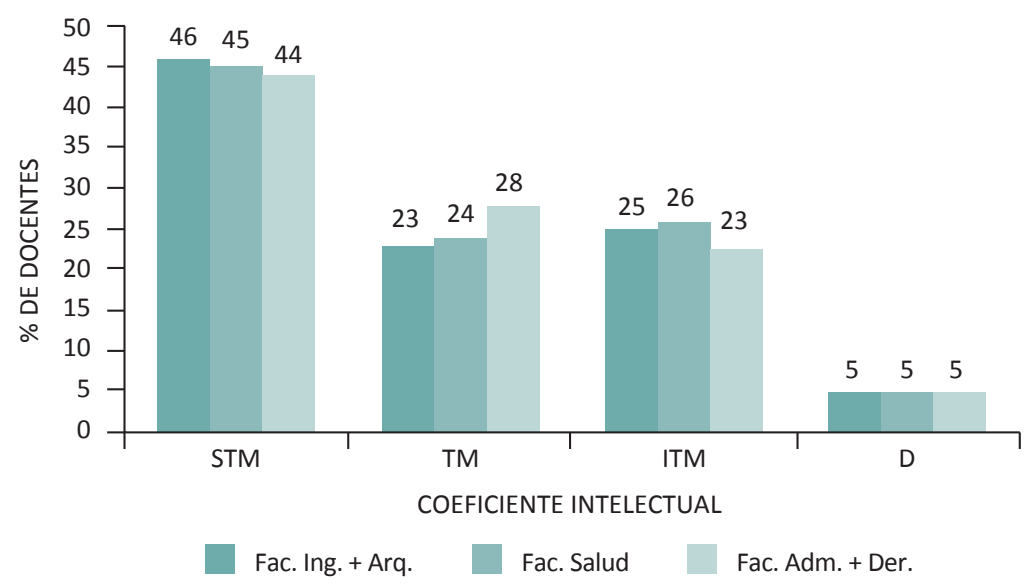

Figura 6.

Fuente propia

En la tabla 7 se presentan los resultados obtenidos por los docentes en el test de Matrices Progresivas Raven escala general; aplicada a los docentes que trabajan por facultad separada, y se resume en lo siguiente:

- Las docentes de la Facultad de Ingenierías y Arquitectura tienen un mayor C.I.STM res- pecto a los docentes de la Facultad de Ciencias de la Salud y a los docentes de la Facultad de Ciencias Administrativas y Derecho en una proporción de $46 \%$, $45 \%$ y $44 \%$ respectivamente.

- Las docentes de la Facultad de Ciencias Administrativas y Derecho tienen un mayor C.I.TM respecto a los docentes de la Facul- 
tad de Ciencias de la Salud y a los docentes de Ingenierías y Arquitectura en una proporción de $28 \%$, $24 \%$ y $23 \%$ respectivamente.

- Las docentes de la Facultad Ciencias de la Salud tienen un mayor C.I.ITM respecto a los docentes de la Facultad de Ingenierías y Ar- quitectura y a los docentes de la Facultad de Ciencias administrativas y Derecho en una proporción de $26 \%$, $25 \%$ y $23 \%$ respectivamente.

- Las docentes de las 03 Facultades tienen un mismo porcentaje de $5 \%$ de docentes con un C.I. D.

\section{d) Comparación del coeficiente intelectual de los docentes por formación}

\section{Tabla 8. Comparación del C.I. de docentes por formación}

\begin{tabular}{lrrrrrrrrr} 
RAVEN & C- ADM.+DER- & $\%$ & C. ING.+ARQ- & $\%$ & C- SALUD & $\%$ & C. EDUC. & $\%$ \\
\hline STM & 17 & 49 & 35 & 50 & 31 & 50 & 21 & 49 \\
TM & 9 & 26 & 17 & 24 & 15 & 24 & 11 & 26 \\
\hline ITM & 7 & 20 & 14 & 20 & 13 & 21 & 9 & 21 \\
D & 2 & 6 & 4 & 6 & 3 & 5 & 2 & 5 \\
\hline & 35 & 100 & 70 & 100 & 62 & 100 & 43 & 100 \\
\hline
\end{tabular}

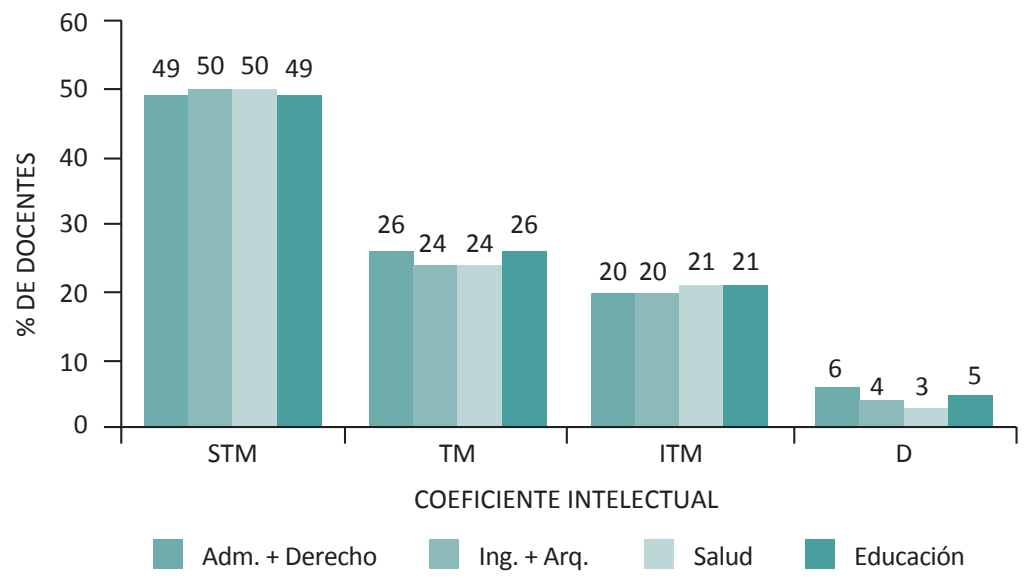

Figura 7.

Fuente propia

En la tabla 8 se presentan los resultados obtenidos por los docentes en el test de Matrices Progresivas Raven escala general; aplicada a los docentes por su formación académica o su carrera por separado y se resume en lo siguiente:

- Las docentes de formación de Ingenierías, Arquitectura y de Salud tienen un mayor número de docentes con C.I.STM con porcentaje de $50 \%$ respecto a los docentes de formación en Ciencias Administrativas, Derecho y Educación con $49 \%$.
- Las docentes de formación de Ciencias Administrativas, Derecho y Educación tienen un porcentaje de $26 \%$ de coeficiente intelectual término medio respecto a los docentes de formación en Ingenierías, Arquitectura y Ciencias de la Salud, con $24 \%$.

- Las docentes de la de formación de Ciencias de la Salud y Educación tienen mayor número de docentes con coeficiente intelectual inferior al término medio con $21 \%$ respecto a los docentes de formación de Ingenierías, Arquitectura y Ciencias Administrativas y Derecho con 20\%. 
- Los docentes de formación de Ciencias Administrativas, Derecho y Educación tienen mayor porcentaje de docentes con coeficien- te intelectual deficiente respecto a los docentes de formación de Salud, Ingenierías y Arquitectura.

\section{e) Comparación del coeficiente intelectual de los docentes por edad}

\section{Tabla 9. Comparación del C.I de docentes por edades}

\begin{tabular}{lrrrrrrrrrr} 
RAVEN & $23-30$ & $\%$ & $31-40$ & $\%$ & $41-50$ & $\%$ & $51-60$ & $\%$ & $61-70$ & $\%$ \\
\hline STM & 10 & 43 & 21 & 49 & 36 & 46 & 21 & 46 & 9 & 45 \\
TM & 3 & 13 & 11 & 26 & 16 & 21 & 5 & 11 & 3 & 15 \\
ITM & 9 & 39 & 9 & 21 & 21 & 27 & 18 & 39 & 7 & 35 \\
D & 1 & 4 & 2 & 5 & 5 & 6 & 2 & 4 & 1 & 5 \\
\hline & 23 & 100 & 43 & 100 & 78 & 100 & 46 & 100 & 20 & 100 \\
\hline
\end{tabular}

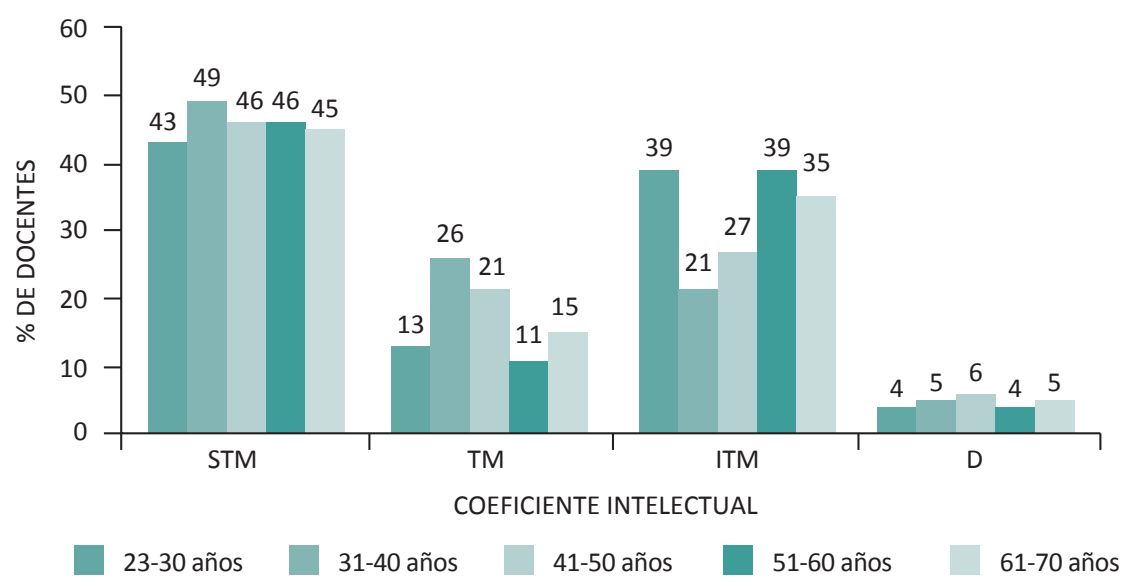

Figura 8.

Fuente: propia

En la tabla 9 se presentan los resultados obtenidos por los docentes en el test de Matrices Progresivas Raven escala general; aplicado a los docentes por edades, separado por décadas que se resume en lo siguiente:

- Las docentes con edades que oscilan de 31 a 40 años tienen un mayor C.I.STM, con porcentaje de $49 \%$ seguido por los docentes de $41-50,51-60$ con $46 \%$ ambos, 61-70 con 45 $\%$ y 23-30 años $43 \%$

- Las docentes con edades que oscilan de 31 a 40 años tienen un mayor C.I.TM, con porcentaje de $26 \%$ seguido por los docentes de 41-50 con $21 \%$, luego los docentes con 61-
70 con $15 \%$, 23-30 años con $13 \%$ y los docentes con 51-60 años de edad con 11\%

- Las docentes con edades que oscilan de 23-30 y 51-60 años tienen un mayor número de docentes con C.I.ITM, con porcentaje de $39 \%$ seguido por los docentes de 61-70 con $35 \%$, luego los docentes con 41-50 años con $27 \%$ y los docentes de 31-40 años de edad con un $21 \%$.

- Las docentes con edades que oscilan de 41 a 50 años tienen un mayor número de docentes con C.I.D, con porcentaje de $6 \%$ seguido por los docentes de 31-40 y 61-70 que tienen el mismo porcentaje de $5 \%$, luego los docentes con 23-30 y 51-60 años que tienen el mismo porcentaje de $4 \%$. 


\section{Análisis e interpretación de la variable: fac- tores institucionales}

\section{Económico}

Las universidades públicas se ciñen a las normas de derecho público, entre ellas la Ley de Presupuesto para el Sector Público Anual y demás disposiciones legales que rigen la materia económica y presupuestal.

Las universidades privadas ejecutan su presupuesto de acuerdo a las normas de derecho privado y su normativa interna.

Las universidades Privadass de Ica (UPI) no tienen un presupuesto asignado para financiar trabajos de investigación, lo que imposibilita la iniciativa de los docentes y estudiantes investigadores.

\section{Condición laboral}

Los docentes de las UPI tienen un contrato a tiempo parcial,; que consiste en permanecer la cantidad de horas que implica cada curso por semana, y no tienen ningún compromiso laboral adicional.

\section{Categoría del docente}

Los docentes de las UPI no tienen definida la categoría

\section{Régimen de dedicación}

Los docentes de las UPI tienen el régimen de tiempo parcial, porque dedican a las tareas académicas un tiempo menor a la jornada legal de trabajo.

\section{Grado académico}

Los docentes de las UPI tienen la siguiente composición:

- Titulado

- Magíster

- Especialista

- Doctor

\section{Profesor investigador}

Profesor investigador es el de categoría extraordinaria que se dedica exclusivamente a la creación y producción intelectual (Ley Universitaria, artículo $50^{\circ}$ ).

La UPI no cuenta con docentes en la categoría extraordinaria

\section{Formación del investigador}

En los últimos años hay una renuencia marcada de parte de los estudiantes egresados de elaboración de tesis para optar al título profesional en el pregrado, dado que la actual normatividad permite realizar otras modalidades para optar al título profesional; motivo por el cual, la titulación mediante la presentación de tesis viene siendo desplazada.

\section{Organizacional}

Los investigadores de la mayoría de las universidades no están trabajando con sus pares tanto a nivel nacional como internacional, motivándose una necesidad de formación de redes.

Algunas de las UPI han creado el Vicerrectorado de Investigación y Postgrado, incluso Instituto de Investigación.

III. Análisis e interpretación de la variable: política de estado en ciencia, tecnología e innovación

El gobierno actual ha definido la política de inversiones en Ciencia Tecnología e Innovación (CTI) 2013-2020, en la que considera necesario realizar esfuerzos para fortalecer las condiciones básicas para el desarrollo de las actividades de CTI, tales como las de recursos humanos y la infraestructura tecnológica y brindar apoyo efectivo a la innovación tecnológica mediante instrumentos que potencien la actividad de las empresas. Asimismo, ha previsto privilegiar acciones orientadas a facilitar la adquisición, transferencia 
y difusión de tecnologías; pone énfasis en actores que están dispuestos y ávidos por mejorar sus capacidades tecnológicas porque hay mercados que los premiarán por sus esfuerzos.

El Perú tiene dentro de la política actual de gobierno incrementar el financiamiento para Investigación y Desarrollo (I+D) hasta el 0,07\% del $\mathrm{PBI}$, en un periodo no mayor a tres años.

\section{DISCUSIÓN DE RESULTADOS}

Los resultados obtenidos en esta evaluación del coeficiente intelectual de los docentes permite confirmar que no todos los docentes de la UPI tienen cualidades de investigador, sin que eso descalifique al profesional que no haya alcanzado el puntaje que requiere para estar dentro del profesor investigador.

También se ha observado que los profesionales de las carreras profesionales de ingenierías y salud se ubican con buenos puntajes, lo que corrobora la teoría de que son estas las carreras que hace falta impulsar en los países en desarrollo si queremos tener ciencia y tecnología; esta es una tesis comprobada por países desarrollados como Estados Unidos, Japón, etc. Sin embargo, se da más prioridad a las administrativas.

Otro de los resultados que revela esta investigación es que los docentes de 31 a 40 años respondieron mejor a esta investigación, dando la impresión de que los años de experiencia pueden ser desplazados por los jóvenes profesionales por el manejo de las novedades de la tecnología como el acceso a la información con más rapidez y el dominio de la tecnología informática, que a los adultos les es algo pesado.

En el análisis, el resultado por el género y la facultad en la que trabaja no es significativo, lo primero porque la inteligencia no tiene sexo, sino de los estímulos externos y el tipo de gen que ha heredado; lo segundo referido en la facultad, se refiere al estimulo que generará el entorno laboral para desarrollar investigación.

En lo que concierne al factor institucional, está claro que no habrá investigación seria si no se satisfacen todos los factores indicados en el análisis.

De igual modo pasa con la variable política de estado específica para el desarrollo de ciencia, tecnología e innovación, puesto que mas allá de la voluntad política tiene que haber decisión de asignación de recursos económicos similares a los países de Latinoamérica que destacan, como el caso de Brasil que destina 1,16\% del PBI mientras que Perú asigna actualmente solo 0,15 \% PBI.

También es necesario hacer notar que el estado solo asigna recursos a las universidades públicas, y también debe considerar a las universidades privadas con algún mecanismo que permita monitorear los resultados concretos de investigación valida y de aplicación objetiva y no distribuir como hoy una bonificación a la investigación, pero de investigación no hay nada.

Los resultados de esta investigación demuestran precisamente que sí es posible focalizar a los docentes con cualidades de investigador, su ubicación laboral, sexo, edad, formación, nivel académico, que ayudaría a orientar los recursos de manera óptima a los docentes que realmente pueden presentar resultados concretos y de utilidad y así dejar el viejo método de distribuir dinero sin la cualificación apropiada, o sea a todos sin distinción alguna, y el resultados es conocido.

\section{CONCLUSIONES}

a) Del análisis de la variable: fenotipo del docente

- De la figura 3 se concluye que la UPI tiene un $50 \%$ de su plana docente con coeficiente 
intelectual (C.I.) superior al término medio (STM).

- Los docentes con grado académico de magíster y/o doctor tienen mayor porcentaje de docentes con coeficiente intelectual superior al término medio con porcentaje de $46 \%$ y titulados $45 \%$, no es significativo, en términos de trabajos de investigación científica, según los resultados estadísticos.

- Los docentes de la Facultad de Ingeniería y Arquitectura tienen mayor número de docentes con coeficiente intelectual superior al término medio con un porcentaje de $46 \%$ respecto a la Facultad de Ciencias de la Salud con $45 \%$ y Facultad de Ciencias Administrativas y Derecho con $44 \%$, es significativo.

- Los docentes de formación en Ingenierías, Arquitectura y Salud tienen mayor número de docentes con coeficiente intelectual superior al término medio con $50 \%$, respecto a los docentes de formación de Ciencias Administrativas, Derecho y Educación con 49 $\%$, es significativo.

- Hay mayor número de docentes de edad 31-40 con coeficiente intelectual superior al término medio con un porcentaje de $49 \%$, seguido por los docentes de 41-50 y 51-60 años de edad con un porcentaje de $46 \%$, luego están los docentes de 61-70 años de edad con $45 \%$ y los docentes de 23-30 años de edad con $43 \%$, es significativo, lo que concluye que los docentes más jóvenes y de más edad tendrían menos aporte a los trabajos de investigación científica.

b) Del análisis de la variable: factores institucionales

- La UPI no tiene asignado un presupuesto para investigación.

- Los docentes de la UPI no tienen definida una categoría.

- Los docentes de la UPI tienen un régimen laboral de tiempo parcial,
- La UPI permite obtener el título profesional bajo la modalidad de cursos especiales de titulación.

c) Del análisis de la variable: Política de Estado en Ciencia, Tecnología e Innovación.

- La política de inversiones en Ciencia Tecnología e Innovación (CTI) 2013-2020 considera necesario realizar esfuerzos para fortalecer las condiciones básicas para el desarrollo de las actividades de CTI, tales como las de recursos humanos y la infraestructura tecnológica y brindar apoyo efectivo a la innovación tecnológica mediante instrumentos que potencien la actividad de las empresas.

- El Perú tiene dentro de la política actual de gobierno incrementar el financiamiento para Investigación y Desarrollo (I+D) hasta el $0,07 \%$ del PBI, en un periodo no mayor a tres años.

\section{REFERENCIAS}

Antunes C. (2005). Inteligencias múltiples. Cómo estimularlas y desarrollarlas. Primera edición. Empresa Editora el Comercio S. A., Perú.

Arquero Avilés, R. y Salvador Oliván, J. A. (2004). Evaluación y análisis de la producción de los autores de trabajos difundidos en publicaciones periódicas editadas en español. Documentación de las Ciencias de la Información, 27: 53-74.

Morales Sosa Flor Elena (2010). Estudio de Salud Mental en Estudiantes Universitarios de la Facultad de Ciencias de la Educación de la Universidad de Carabobo. Madrid, noviembre 2010

Frías, J. A. Y Romero Gómez, P. (1998). “¿Quiénes son y qué citan los investigadores que publican en las revistas españolas de Biblioteconomía y Documentación?", en Anales de Documentación, 1:29-53.

Gorbea Portal, S. (2005), Modelo teórico para el estudio métrico de la información documental. Gijón: Trea. 
Jiménez Contreras, E. Y Moya Anegón, F. (1997). "Análisis de la autoría en revistas españolas de Biblioteconomía y Documentación”, 1975-1995, en Revista Española de Documentación Cientifica, 20(3):252-267.

Kim, S. D. Y Kim, M. T. (1979), "Academic library research: a twenty year perspective”, en Stueart, R. D. y Johnson, R. D. New horizons for academic libraries, New York: K. G. Saur, pp. 375-383.

Díaz Romero Yosvani, Nueva Educación para Nuevo Milenio. Secretaría Ejecutiva de la OCLAE, Cuba.

Bermúdez García Javier E. Investigación cientifica en el Perú: factor critico de éxito para el desarrollo del país.

Carazo, Mercedes Inés, Hurtado, Ángel P. (2010). Estrategia nacional de desarrollo de la innovación y la productividad en el Perú: elementos para una propuesta. Lima, Ministerio de Industria, Turismo, Integración y Negociaciones Comerciales Internacionales (MITINCI), 2000.

Deza Rivasplata, Jaime (2013). Metodología de la investigación cientifica. Cuarta edición. Dirección de Investigación de la Universidad Alas Peruanas, Lima.

Latorre, Víctor. (2003). Capacidades para la investigación cientifica: las ciencias básicas en el Perú. Lima, BID.
Tarnawiecki, Carlos Sánchez. (2003). Capacidades de investigación cientifica y tecnológica en el Perú en el área temática de tecnologias de información y comunicaciones. Lima, Consejo Nacional de Ciencia y Tecnología (CONCYTEC).

UNESCO.(2007) A Global Perspective On Research And Development. París, Institute for Statistics.

Mullin, James. (2002). Análisis del sistema peruano de innovación. Una contribución al desarrollo del Programa de Ciencia y Tecnología. BID/Perú.

Watson, P. D. (1985) "Production of scholarly articles by academic librarians and library school faculty", en College \& Research Libraries, 46(4):334-342.

Asamblea Nacional de Rectores, Instituto Nacional de Estadística e Informática (2010). II Censo Nacional de Universitario-2010.

Ramos Jaubert Rocío Isabel, García Santos Zoila Libertad, Ramírez Chávez Jorge. Factores y caracteristicas del investigador.

ANR. Secretaría Ejecutiva y la Dirección de General de Investigación Panorama de la investigación en la Universidad del Perú, año 2012.

MEF. Dirección General de Políticas de Inversión. Politica de inversión pública en Ciencia, Tecnología e Innovación, prioridades 2013-2020. 